Europäische Zulassung von Eltrombopag für chronische Immunthrombozytopenie empfohlen

\section{Positive Opinion des CHMP für ersten oralen Thrombopoietin-Rezeptor- Agonisten erteilt}

Der Ausschuss für Humanarzneimittel (CHMP) der Europäischen Arzneimittelzulassungsbehörde EMEA hat die Zulassung von Eltrombopag (geplanter Handelsname Revolade ${ }^{\circledR}$ ) empfohlen. Diese so genannte «Positive Opinion» gilt für die orale Behandlung der Thrombozytopenie bei Erwachsenen mit chronischer Immunthrombozytopenie (ITP) nach Splenektomie, die auf die üblichen Behandlungen mit Corticosteroiden und/oder Immunglobulinen nicht oder nicht mehr ansprechen. Außerdem kann Eltrombopag als Second-Line-Therapie in Erwägung gezogen werden, wenn eine Splenektomie nicht angezeigt ist.

Die Positive Opinion des CHMP gründet sich auf 2 randomisierte, doppelblinde, Placebokontrollierte Phase-III-Studien (TRA100773B [1] und RAISE [2]) sowie 2 offene Studien (REPEAT [3] und EXTEND [4]). Alle Studien wurden bei Erwachsenen durchgeführt, die bereits wegen ihrer ITP vorbehandelt waren. Die Ergebnisse zeigten, dass Eltrombopag im Vergleich zu Placebo die Blutplättchenzahl signifikant erhöhte, das Auftreten von Blutungen verminderte und die Lebensqualität verbesserte $[2,4,5,6]$. Darüber hinaus konnte bei vielen Patienten unter Eltrombopag eine zusätzliche ITP-Medikation, meist Steroide, reduziert oder ganz abgesetzt werden [2].

Eltrombopag erwies sich in den klinischen Studien als gut verträglich $[1,2,7]$.

\section{Referenzen}

Bussel JB et al.: Lancet 2009;373:641-8.

2 Cheng G et al.: Blood 2008;112:400. (ASH Annual Meeting Abstracts).

3 Psaila B et al.: 13th Congress of the European Haematology Association, Juni 2008; abstr 0294.

4 Saleh et al.: Blood 2009;114(22); abstr 682

5 Bussel et al.: Haematologica 2009;94(S 2); abstr 239.

6 Provan et al.: Haematologica 2009;94(S 2); abstr 233.

7 Bussel JB et al.: N Engl J Med 2007;357:2237-47.

Weitere Informationen bei

GlaxoSmithKline GmbH \& Co. KG

Senior-PR-Manager

Dr. Anke Helten

Theresienhöhe 11, 80339 München

Tel. +49 89360448102

anke.helten@gsk.com

\section{Operationen bei Hämophilie-Pati- enten: hohe Qualitäts- und Sicher- heitsstandards}

Die speziellen Therapieoptionen in Zusammenhang mit elektiven operativen Eingriffen bei Hämophilie-Patienten waren Themen eines von Novo Nordisk Pharma unterstützten Satellitensymposiums im Rahmen der 54. Jahrestagung der Gesellschaft für Thrombose- und Hämostaseforschung (GTH) 2010 in Nürnberg.

Aktuelle Hinweise zur Dosierung in Abhängigkeit von der Art und Größe des Eingriffs finden sich für Deutschland in den «Querschnitts-Leitlinien zur Therapie mit Blutkomponenten und Plasmaderivaten» der Bundesärztekammer (2009) [1] und international in einer ReviewArbeit [2] von Experten aus europäischen Hämophiliezentren. Letztere enthalten Angaben zu prä- und postoperativen Faktor-Zielkon- zentrationen, Dauer der Substitutionstherapie und sonstigen hämostaseologischen Therapiemaßnahmen für elektive Eingriffe bei hämophilen Patienten.

Bei Hämophilie-Patienten sind die Blutungen in einem Großteil der Fälle in Gelenken lokalisiert. Laut Prof. Thomas Wallny (Kamp-Lintfort) weisen hämophile Patienten, die vor der Ära der Heimselbstbehandlung geboren wurden, im Allgemeinen mindestens ein durch Blutungen stark geschädigtes Gelenk auf. Rezidivierende Blutungen können schwere Gelenkveränderungen verursachen, die häufig zu einer sekundären Arthrose mit fortschreitenden Fehlstellungen und Gelenkversteifungen führen. Die Folgen sind letztlich weitreichende Einschränkungen der Mobilität und der damit verbundenen Lebensqualität. Verschiedene orthopädische Operationen dienen dazu, die Folgen von Gelenkblutungen zu mildern.

\section{Quelle}

Satellitensymposium «Elektive chirurgische Eingriffe be Hämophilie-Patienten», GTH Kongress 2010, 24. Februa 2010, Nürnberg; Veranstalter: Novo Nordisk Pharma GmbH

\section{Referenzen}

1 Querschnittsleitlinien zur Therapie mit Blutkomponenten und Plasmaderivaten, Bundesärztekammer (Hrsg), 4. erweiterte Auflage. Köln, Deutscher Arzte-Verlag, 2009.

2 Hermans C et al. Haemophilia 2009;15(3);639-658.

Weitere Informationen bei MEDIZIN \& MARKT GmbH

Franziska Thiele

Dachauer Straße 36-38, 80335 München

Tel. +498938393022

thiele@medizin-und-markt.de

\title{
Ticker+++ Ticker+++ Ticker+++ Ticker+++ Ticker+++ Ticker+++ Ticker+++
}

CSL Behring. Seit einem Jahr ist Privigen ${ }^{\circledR}$, ein neuartiges polyvalentes intravenöses Immunglobulin, auf dem Markt. Es ist ab sofort auch als Mehrfachpackung (N2) in den Größen 3×10 und $3 \times 20 \mathrm{~g}$ erhältlich. Damit reduziert sich die Zuzahlungsleistung der Patienten um 2 Drittel. Für eine höhere Flexibilität in Praxen und Kliniken wurde zudem eine kleine 2,5-g-Packung in das Produktportfolio aufgenommen.

CSL Behring

Dr. Peer Lotichius

Tel. +4969305171 02

peer.lotichius@cslbehring.com
Genzyme. In der Studie CAM314 zeigte sich, dass bei den Patienten mit rezidivierender bzw. refraktärer chronischer lymphatischer Leukämie, die FluCAM (MabCambath ${ }^{\circledR}$ + Fludara ${ }^{\circledR}$ ) erhielten, das Risiko für Krankheitsprogression oder Tod signifikant geringer war als bei alleiniger Gabe von Fludara. Angesichts der positiven Interimsergebnisse beabsichtigt Genzyme jetzt, die Erweiterung der Zulassung von MabCampath um diese Kombinationstherapie zu beantragen.

Genzyme GmbH

Dr. Paul Fabry

Tel.: +49 61023674620

paul.fabry@genzyme.com
Novartis. Eisenüberladung bei myelodysplastischen Syndromen (MDS): Eine aktuelle Analyse des Düsseldorfer MDS-Registers belegt eine signifikante Verlängerung des medianen Gesamtüberlebens, wenn MDS-Patienten mit Eisenüberladung eine Eisenchelat-Therapie erhalten. Die mediane Gesamtüberlebenszeit in der Eisenchelat-Therapie-Gruppe betrug 75 Monate vs. 49 Monate in der Gruppe ohne Chelation $(p=0,002)$.

Novartis Pharma GmbH

Dr. Irene Roth

Tel. +49 9112730

irene.roth@novartis.com 